\title{
Three-dimensional MHD modeling of vertical kink oscillations in an active region plasma curtain ${ }^{\star}$
}

\author{
L. Ofman ${ }^{1,2,3}$, M. Parisi ${ }^{4,5}$, and A. K. Srivastava ${ }^{6}$ \\ 1 Catholic University of America, Washington, DC 20064, USA \\ 2 NASA Goddard Space Flight Center, Code 671, Greenbelt, MD 20771, USA \\ e-mail: Leon.0fman@nasa.gov \\ 3 Visiting, Department of Geophysics and Planetary Sciences, Tel Aviv University, 69978 Tel Aviv, Israel \\ ${ }^{4}$ Department of Mechanical and Aerospace Engineering, Sapienza University of Rome, via Eudossiana 18, 00184 Rome, Italy \\ 5 Presently at Department of Earth and Planetary Sciences, Weizmann Institute of Science, 234 Herzl Steet, 7610001 Rehovot, Israel \\ ${ }^{6}$ Department of Physics, Indian Institute of Technology (Banaras Hindu University), 221005 Varanasi, India
}

Received 25 September 2014 / Accepted 8 August 2015

\begin{abstract}
Context. Observations on 2011 August 9 of an X 6.9-class flare in active region (AR) 11263 by the Atmospheric Imaging Assembly (AIA) on board the Solar Dynamics Observatory (SDO), were followed by a rare detection of vertical kink oscillations in a large-scale coronal active region plasma curtain in extreme UV coronal lines with periods in the range 8.8-14.9 min.

Aims. Our aim is to study the generation and propagation of the magnetohydrodynamic (MHD) oscillations in the plasma curtain taking the realistic 3D magnetic and the density structure of the curtain into account. We also aim to test and improve coronal seismology for a more accurate determination of the magnetic field than with the standard method.

Methods. We use the observed morphological and dynamical conditions, as well as plasma properties of the coronal curtain, to initialize a 3D MHD model of the observed vertical and transverse oscillations. To accomplish this, we implemented the impulsively excited velocity pulse mimicking the flare-generated nonlinear fast magnetosonic propagating disturbance interacting obliquely with the curtain. The model is simplified by utilizing an initial dipole magnetic field, isothermal energy equation, and gravitationally stratified density guided by observational parameters.

Results. Using the 3D MHD model, we are able to reproduce the details of the vertical oscillations and study the process of their excitation by a nonlinear fast magnetosonic pulse, propagation, and damping, finding agreement with the observations.

Conclusions. We estimate the accuracy of simplified slab-based coronal seismology by comparing the determined magnetic field strength to actual values from the 3D MHD modeling results, and demonstrate the importance of taking more realistic magnetic geometry and density for improving coronal seismology into account.
\end{abstract}

Key words. magnetohydrodynamics (MHD) - Sun: corona - Sun: magnetic fields - Sun: oscillations - Sun: flares - Sun: activity

\section{Introduction}

With the launch of the Solar Dynamics Observatory (SDO), the detection of waves in extreme ultraviolet (EUV) emission in the corona using the Atmospheric Imaging Assembly (AIA) instrument became possible in many observed events with potential applications to coronal seismology (CS), and to understand the physical conditions in the corona. Using SDO/AIA EUV channels, transverse coronal loop oscillations were observed to be ubiquitous in active region loops (McIntosh et al. 2011) and were analyzed in great detail with application to coronal seismology (e.g., Aschwanden \& Schrijver 2011; Yuan \& Nakariakov 2012; White \& Verwichte 2012; Wang et al. 2012; Nisticò et al. 2013, 2014; Verwichte et al. 2013b,a). Nevertheless, vertical oscillations in coronal loops or magnetic arcades are rarely observed in EUV emissions. This is the case in spite of recent high-resolution and high-cadence observations by AIA, and past high-resolution observations by the Transition Region and Coronal Explorer (TRACE) satellite. The vertical kink oscillations of a curved coronal loop are fundamentally different from

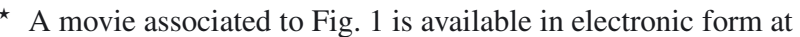
http: //www. aanda.org
}

the horizontal kink oscillations, since they are confined to the loop curvature plane and can lead to a change of loop length. Horizontal kink oscillations were first detected by Aschwanden et al. (1999) and Nakariakov et al. (1999) and have been studied extensively since then in many papers motivated by their potential use in magnetohydrodynamic (MHD) CS for localized coronal structures (e.g., reviews by Nakariakov \& Verwichte 2005; Andries et al. 2009; De Moortel \& Nakariakov 2012; Liu \& Ofman 2014, and references therein). However, later Wang \& Solanki (2004) reported the first confirmed detection of vertical oscillations in a coronal loop using TRACE $195 \AA$ Abservations of a coronal loop on the limb on 17 April 2002, with additional cases analyzed in Wang et al. (2008). Vertical oscillations in a hot coronal loop following a flare/CME was reported for the first time by White et al. (2012) using SDO/AIA $131 \AA$ ( 10 MK) and $94 \AA$ ( 6.3 MK) EUV bandpasses. Vertical oscillations in cool coronal loops were also detected with SDO/AIA in conjunction with STEREO/EUVI. These oscillating loops were triangulated using STEREO/EUVI data, and oscillation properties derived from SDO/AIA and STEREO/EUVI were utilized for refined coronal seismology (Aschwanden \& Schrijver 2011). Recently, Srivastava \& Goossens (2013, hereafter SG13), 

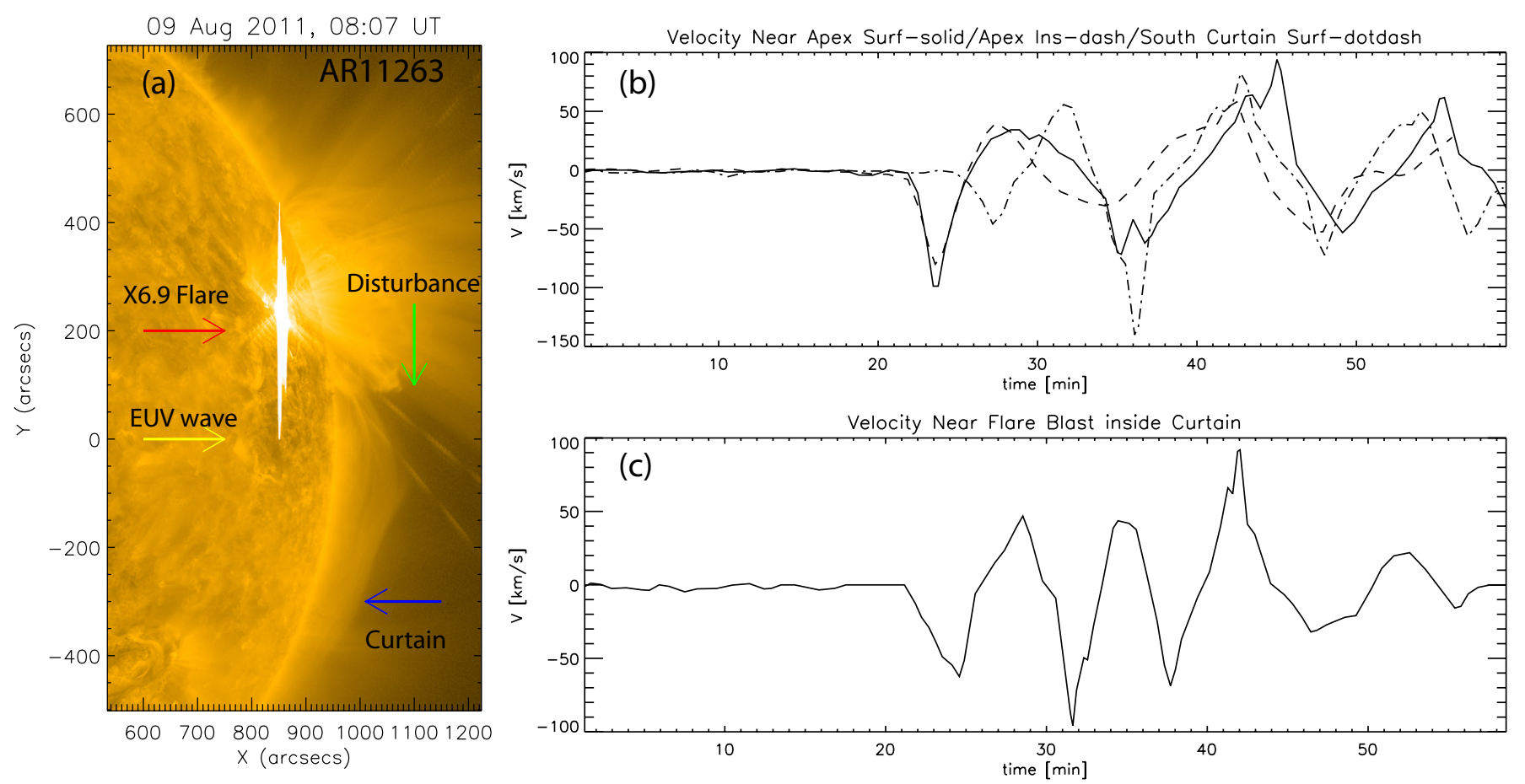

Fig. 1. a) Snapshot from 9 August 2011 at 08:07 illustrates the X 6.9 flare (red arrow), EUV wave (yellow arrow), plasma curtain (blue arrow), and disturbances passing in a narrow channel above it (green arrow) (adapted from SG13). The temporal evolution of is available as a movie in the online edition. b) Temporal evolution of the velocity in various parts of the plasma curtain as observed by SG13. c) Temporal evolution of the velocity near the flare site.

have reported vertical oscillations in a coronal active region plasma curtain observed by SDO/AIA in the cool coronal line of $171 \AA(\sim 1 \mathrm{MK})$. Vertical transverse oscillations were also observed recently in a coronal magnetic flux rope using SDO/AIA (Kim et al. 2014).

While several 2D numerical and analytical models of vertical kink oscillations in the coronal structures have been published (e.g., Selwa et al. 2005, 2006; Verwichte et al. 2006; Gruszecki et al. 2006; Selwa et al. 2007), full three-dimensional MHD modeling is required to study the complete interaction between the excitation mechanisms and the 3D mode polarizations of the oscillations in curved magnetic field geometry and the impulsively excited propagating nonlinear fast magnetosonic waves that are launched by a flare that induces the oscillations. The 3D MHD modeling of bipolar active region (AR) loop oscillations provides further insight into the physical scenario and inherent cause for the somewhat rare detection of this phenomena (Selwa et al. 2011b). At present, the application of coronal seismology is often based on a linear dispersion relation, for waves in cylindrical slabs or otherwise simplified structures, which compromises the accuracy of the method (e.g., Ofman 2009; Pascoe \& De Moortel 2014). To improve CS, one needs to apply more realistic modeling of the oscillating structures. A more realistic, bipolar magnetic field (e.g., Miyagoshi et al. 2004; McLaughlin \& Ofman 2008; Selwa \& Ofman 2009; Selwa et al. 2011a,b, 2013; Pascoe \& De Moortel 2014) or an extrapolated magnetogram based magnetic field (e.g., Ofman 2007; Schmidt \& Ofman 2010) and gravitationally stratified density structures were modeled with 3D MHD to study waves. These studies demonstrate the advantage of considering 3D realistic magnetic field topology in the unambiguous determination of seismologically derived coronal magnetic field.

In the present article, we utilize recent observations by SG13 of vertical oscillations of a coronal curtain to set up the parameters of the 3D MHD model. For the first time, we study the excitation, propagation, and damping of vertical oscillations in a coronal bipolar curtain, with applications and quantitative tests of coronal seismology by comparing these observations to 3D MHD modeling results. The goal of this study is to demonstrate the significant improvement of coronal seismology by applying more realistic magnetic field topology and density modeling in 3D MHD calculation, rather than to reproduce an actually observed active region in detail. The paper is organized as follows: in Sect. 2, we present a brief summary of the observations, the numerical model is described in Sect. 3, the numerical results are in Sect. 4, and Sect. 5 is devoted to the discussion and conclusions.

\section{Summary of the observations}

Recently, the detection of a diffused and laminar plasma curtain at the western equatorial corona, which oscillates vertically during passage of the coronal disturbances (EUV waves) triggered by an X 6.9 class solar flare (start: 07:48 UT; peak: 08:05 UT; end: 08:08 UT) in AR 11263 (N17 W69) in its vicinity on 9 August 2011, was reported and analyzed by SG13 (cf. Fig. 1a). The vertical kink oscillations are triggered near the flare energy release site within the curtain $(8.8 \mathrm{~min})$, in the middle both at the apex (14.9 $\mathrm{min})$ and inside (13.3 $\mathrm{min})$, as well as at the surface in the southernmost part of the curtain $(12.7 \mathrm{~min})$. The properties of these excited waves strongly depend on the local plasma and magnetic field conditions of the plasma curtain. The right panels of Fig. 1 show the temporal evolution of the velocity in the middle at the surface (Fig. 1b: solid line), in the inner part (Fig. 1b: dashed line), as well as at the surface in the southward part (Fig. 1b, dotted-dashed line) of the curtain, and near the flare blast site (Fig. 1c). These velocity oscillations in the plane of the sky are completely localized in space at various parts of 
the curtain and derived here from the time-distance measurement data of SG13. The animation of the oscillation observed with SDO/AIA $171 \AA$ A channel is available online.

\section{The 3D MHD numerical model}

We adapt the numerical model of a bipolar AR initially developed by Ofman \& Thompson (2002), and used extensively to study magnetosonic waves in AR loops (e.g., McLaughlin \& Ofman 2008; Selwa \& Ofman 2009; Selwa et al. 2011a,b; Ofman et al. 2012a; Wang et al. 2013). It is not our goal to model the detailed magnetic, density, or thermal structure of the curtain. By using this model, we aim to improve the MHD seismology of the observed vertical kink oscillations with more realistic field and density structure than was used previously in CS based on wave dispersion from simplified slab geometry. The improvement is achieved by matching the parameters of the global bipolar structure of the curtains' field and the gravitational density stratification to the observationally deduced parameters. The parameters of the model were adjusted such that the idealized model is in good agreement with the dynamics of oscillating laminar and diffused large-scale plasma curtain due to the X 6.9 class flare generated disturbances reported by SG13. In particular, the aspect ratio of the dipole was selected to fit the observed active region curtain aspect ratio. The temperature that determines the gravitational scale height of the density was selected to match the observed line emission temperature, the normalizing Alfvén speed, and the parameters of the velocity Gaussian pulse launched at the boundary were optimized through parametric study to match the observed wave amplitude; we provide further details below.

In order to model the event, we solve numerically the time-dependent, nonlinear, finite- $\beta$, resistive 3D MHD equations with gravity, using a Cartesian grid in the range $(-3.5,3.5) \times$ $(-3.5,3.5) \times(1.0,6.0)$, in normalized units (the normalizations are given below). The adopted method for integrating the partial differential equations is a modified Lax-Wendroff method with fourth-order stabilization term (see Ofman \& Thompson 2002). Furthermore, we use Powell (1994) method to correct for any numerical non-zero divergence of the magnetic field. The set of equations includes the contribution from gravity and the energy equation is eliminated with the isothermal approximation (polytropic index $\gamma=1$ ). Using $\gamma=5 / 3$ would be appropriate for thermodynamic equilibrium conditions not applicable in the corona or when the coronal heating terms and losses are known in detail and computed in the energy equation. However, the polytropic (isothermal) approximation is based on the reasonable approximation that the heating and losses terms are balanced exactly in the energy equation, and combined with the high coronal heat conduction that leads to a constant temperature. The nearly isothermal structure of coronal loops in quiescent active regions has been deduced from past observations (e.g., Aschwanden 2005).

Moreover, observational evidence of coronal loop oscillations shows that the effective polytropic index is close to unity (Van Doorsselaere et al. 2011), in line with our choice of polytropic index. This approximation is also supported by the DEM analysis of the present structure, which only shows about $15 \%$ variability of the temperature in the active region curtain, approximately the observational uncertainty (Srivastava et al., in prep.). Since in the oscillations in the curtain are observed for about $40 \mathrm{~min}$, the effects of resistive dissipation can be neglected because the resistive dissipation time is much longer in both the observed and the modeled structure. At the end of the oscillations, the intensity structure of the curtain reverts mostly to the original form, indicating a negligible net effect of the oscillations on the curtain. Nonisothermal (cooling) loop oscillations were studied in the past (e.g., Morton \& Erdélyi 2009; Erdélyi et al. 2011; Ruderman 2011; Al-Ghafri et al. 2014). However, we neglect the effect of cooling, since the observed active region temperature remains nearly constant on the oscillation timescale.

Multiple processors (up to 144) are used to execute the code, which is parallelized using Message Passing Interface (MPI). We assume that the magnetic field perturbed by the flare can be reasonably described by perturbing the potential dipole (Fig. 2a) in the 3D computational domain, modeled on a $512 \times 512 \times 434$ grid. The high resolution well resolves the background density and magnetic field gradients, as well as the propagating fast magnetosonic pulse launched to model the effects of the flare, and minimizes the possible effect of numerical dissipation; this was tested by comparison with lower resolution runs. The footpoints of the dipole are located at $( \pm 2.5,0.0$, $1.0)$ in the box of normalized units, and this geometry allows us to preserve the aspect ratio of the observed plasma curtain ( $\sim 530 \mathrm{Mm}$ length and height of $\sim 100 \mathrm{Mm}$ ).

The MHD equations are normalized using the following parameters: length scale of $a=R_{\mathrm{S}} / 10$ where $R_{\mathrm{S}}$ is the solar radius, magnetic field at the footpoints $B_{0}=23 \mathrm{G}$; temperature $T_{0}=1 \mathrm{MK}$, which is the isothermal temperature in the model active region curtain; and particle density $n_{0}=10^{9} \mathrm{~cm}^{-3}$ at $z_{\min }=1$. These values are guided by the observationally determined values obtained by SG13 and determine the Alfvén speed of $V_{\mathrm{A} 0}=1586.5 \mathrm{~km} \mathrm{~s}^{-1}$, the Alfvén time $\tau_{\mathrm{A}}=44.1 \mathrm{~s}$, and the isothermal sound speed of $C_{\mathrm{s}}=\left(p_{0} / \rho_{0}\right)^{1 / 2}=129 \mathrm{~km} \mathrm{~s}^{-1}$.

The observed oscillations shown in Fig. 1, and modeled here, are for plasma emitting from Fe IX at $171 \AA$ in EUV. Since the peak response of the $171 \AA$ EUV filter is around $1 \mathrm{MK}$ (Lemen et al. 2012), the observed oscillating magnetic structure shows $1 \mathrm{MK}$ plasma component in the multithermal coronal plasma, and justifies the choice of temperature for the numerical model of the present observation. The selected value for the Alfvén speed exceeds the value estimated by SG13 using simplified (slab) coronal seismology by about $20 \%$. We found, in fact, that we need to adopt a larger value by increasing the background magnetic field $B_{0}$ to match the observed oscillation amplitude and period. We are modeling MHD oscillations of a complex curved magnetic structure, such as a coronal curtain, using an idealized model for the AR based on a dipole magnetic field, thus it is expected that the model values of the physical parameters improve on a simplified slab model, but may still differ from the actual values.

The initial density profile is assumed to be gravitationally stratified and characterized by a normalized scale height $H$, i.e.,

$\rho=\rho_{0} \mathrm{e}^{\left[1 /\left(10+z-z_{\min }\right)-1 / 10\right] / H}$,

where $H=2 k_{\mathrm{B}} T_{0} R_{\mathrm{s}} /\left(10 G M_{\mathrm{s}} m_{\mathrm{p}}\right), k_{\mathrm{B}}$ is Boltzmann constant, $T_{0}$ is the temperature, $G$ is the universal gravitational constant, $M_{\mathrm{S}}$ is the solar mass, and $m_{\mathrm{p}}$ is the proton mass. The initial density distribution is shown in Fig. 2a, as a cut in the $x z$ plane $(y=0)$, where $x$ is the direction of the initial injection of the idealized disturbance that models the effects of the flare (see below). Some closed magnetic field lines that belong to the curtain in this plane are shown. Although the image in Fig. 1a shows higher emission in the curtain than in the corona above this could be the result of somewhat different temperature and decreased plasma density above the AR. We assume that this transition has negligible effect on the oscillation inside the curtain because of the rapid 
(a)

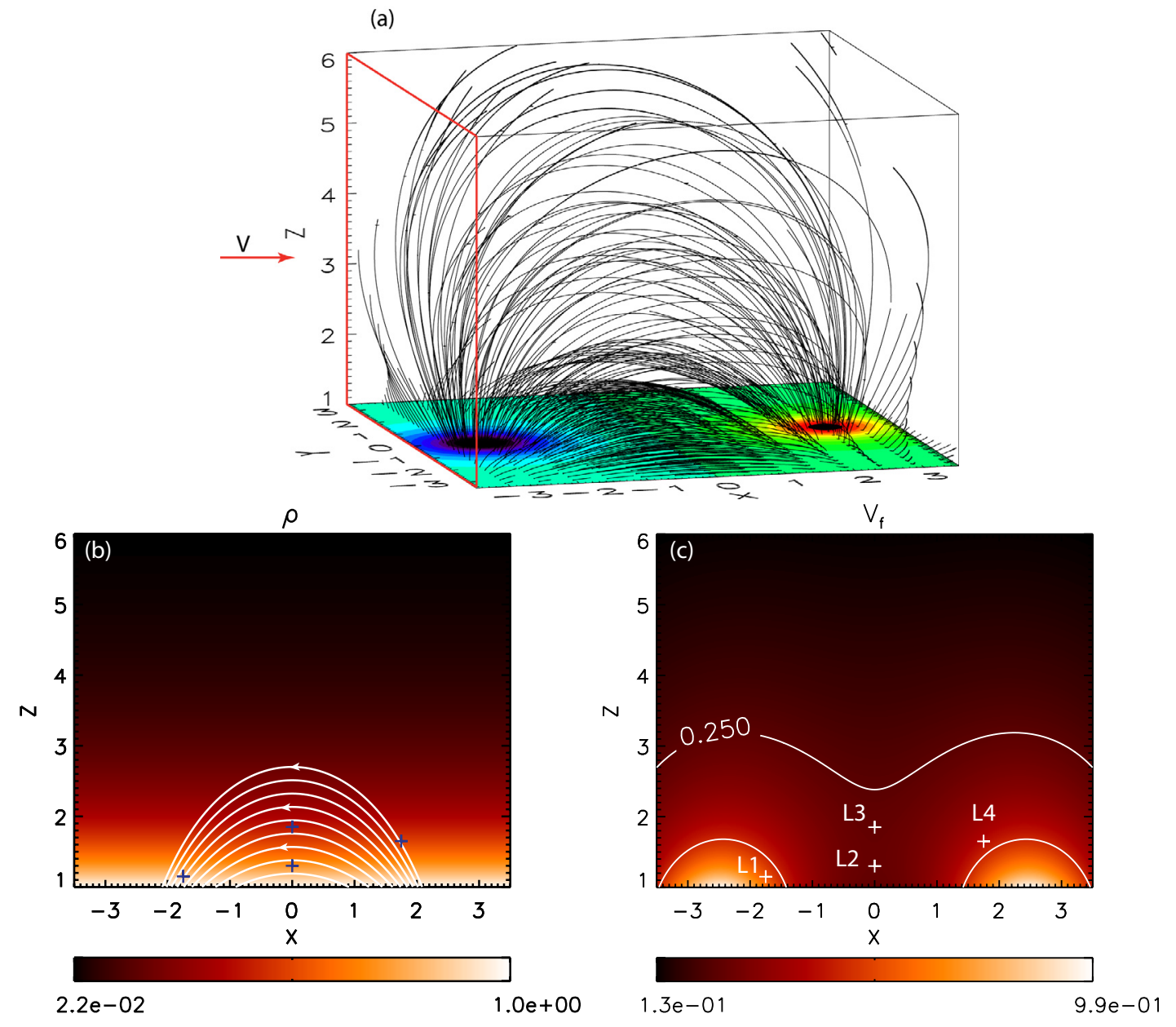

Fig. 2. a) Idealized model of the AR as a magnetic dipole. The red box shows the $y_{z}$ boundary plane at $x=0$ impacted by the propagating disturbance that models the effect of the flare on the active region curtain. The red arrow shows the direction of the impacting velocity pulse applied as the time-dependent boundary condition on this plane. The orientation of the model is turned by $90^{\circ} \mathrm{CCW}$ with respect to the observation shown in Fig. 1. Bottom: normalized initial density distribution overlayed with some of the closed field lines that belong to the curtain are shown (b) and initial fast magnetosonic speed (c) in the $x z$ plane at $y=0$. The blue (white) " + " symbols mark the locations of the points L1-L4 used for temporal evolution diagnostic (see below).

decrease of the magnetic field strength with height for a bipolar magnetic field. In our model the geometry of the curtain is modeled by the dipole field and the (nearly) frozen-in plasma is carried by the field lines during the oscillations. Moreover, our analysis is focused on the regions inside the curtain, with an observationally guided height obtained from the magnetic loops, aspect ratio. The small effect of reflection or refraction on the transverse waves due to the interface region is not considered.

In Fig. 2b, we show the normalized fast magnetosonic speed (in terms of $\left.V_{\mathrm{A} 0}\right), V_{\mathrm{f}}(x, z)=\left[V_{\mathrm{A}}(x, z)^{2}+C_{\mathrm{s}}^{2}\right]^{1 / 2}$ for perpendicular propagation in the $x z$ plane for $y=0$, of the initial state. As it is clear from the image, $V_{\mathrm{f}}$ speed decreases extremely fast with the distance from the footpoints, reducing to $25 \%$ of the maximum value at about just half the height of the grid $(z=3.5)$. This is because, for $C_{\mathrm{s}} \ll V_{\mathrm{A}}$, as we can reasonably assume in this case, $V_{\mathrm{f}}$ behaves basically as $V_{\mathrm{A}}$, thus decreasing as $B \sim 1 / r^{3}$ (if far enough from the dipole) and being inversely proportional to $\rho^{1 / 2}$. The density, which, in turn, decreases nearly exponentially with $z$ and is characterized by a scale height $H$ that depends upon the observationally guided choice of the initial temperature of the coronal plasma in the curtain. The location of points L1-L4, used for a temporal evolution diagnostic are marked with "+" symbols in the $x z$ plane, where the $3 \mathrm{D}$ coordinates in normalized units of the points are $L 1=(-1.75,-3.35,1.15), L 2=(0.0,1.7,1.3), L 3=$ $(0.0,1.7,1.85), L 4=(1.75,-0.9,1.65)$. The choice of these points is consistent with the observing points in SG13 shown in Fig. 1. In the present models, the four locations L1-L4 are inside the oscillating active region curtain, since in the present model we use the length and the corresponding height of the fieldlines in the 3D dipole structure to define the curtain interior. Furthermore, we assume that the region above the curtain has negligible influence on the oscillations of the curtain because of the rapidly decreasing magnetic field strength with height.

Boundary conditions are imposed on each surface at the edges of the 3D computational box. Open boundaries with zero-order extrapolation for every plasma perturbed variable are placed at all boundaries except for the lower boundary $\left(z=z_{\min }\right)$, where we impose a condition of wave reflection simulating linetied boundary, and for the interface between the integration domain and the external disturbances. Since modeling the flare is beyond the scope of the present study, in order to trigger the vertical kink oscillations in the plasma curtain as observed by SG13, we perturb the AR initial state with a Gaussian-shaped velocity pulse applied as a time-dependent boundary condition for short duration compared to typical normal mode periods (similar in 

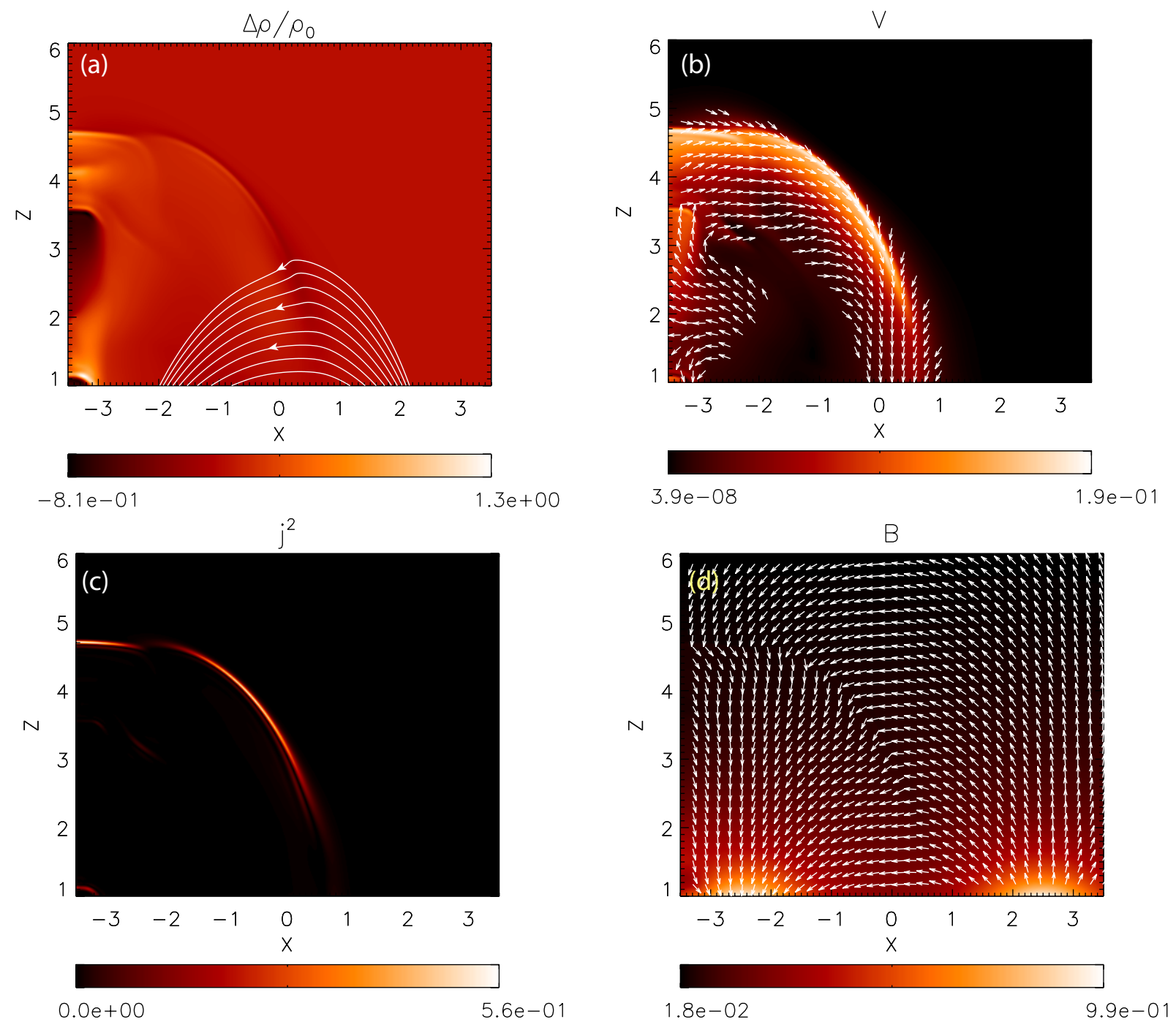

Fig. 3. Snapshot of the cuts in the $x z$ plane at the center of the curtain obtained with the 3D MHD model at $t=14.7 \tau_{A}$, showing the propagating fast magnetosonic disturbance produced by the boundary pulse that models the effects of the flare. a) Perturbed density $\Delta \rho / \rho(t=0)$. Field lines associated with the active region curtain are shown. The distortion of the field lines by the propagating fast magnetosonic disturbance is evident. b) Velocity magnitude and the direction (arrows are shown for $|v|$ greater than $6 \%$ of the absolute maximal velocity). c) Current density map $j^{2}$. d) Magnitude of the magnetic field and the direction. The results show the effects of the initial induced wave pulse and the response of the curtain.

form to those used in Selwa \& Ofman 2009; Selwa et al. 2011a) in the $x$ direction (see Fig. 2a)

$V_{x}=A_{\mathrm{V}} \exp \left[-\left(\frac{z-z_{\min }}{w_{z}}\right)^{2}\right] \exp \left[-\left(\frac{t-\left(t_{1}+2 \delta t\right)}{\delta t}\right)^{2}\right]$,

applied at the $y z$ plane at $x=x_{\min }$ for the duration of the pulse $\delta t=t_{2}-t_{1}=2 \tau_{\mathrm{A}}$. The pulse magnitude is normalized in units of $V_{\mathrm{A} 0}$, and the parameters are $A_{\mathrm{V}}=0.18, w_{z}=0.7$, where $t_{1}$ is the pulse start time at $0.5 \tau_{\mathrm{A}}$ after the model initiation time $t=0$. The value of $w_{z}$ provides the width of the Gaussian pulse in $z$ and deposits the energy in the lower part of the active region curtain, consistent with observations. The values of the pulse parameters have been chosen by performing a parametric study that best fits the observed wave amplitude at point L1-L2. For example, we have repeated the same model runs with several values of $A_{\mathrm{V}}$ in the range $A_{\mathrm{V}}=0.05$ to $A_{\mathrm{V}}=0.25$, and found through interval halving of this parameter that $A_{\mathrm{V}}=0.18$ provides oscillation amplitudes that agree well with the observations. However, the periodicity and the damping rate of the waves are not sensitive to the choice of the pulse parameters for small $\delta t$ compared to the periods. The value of $B_{0}$ were varied in several runs in the range 19-26 G, and as expected, it was found that the oscillation period is proportional to $B_{0}$ with other parameters fixed, providing best fit for $B_{0}=23 \mathrm{G}$.

\section{Numerical results}

In this section, we highlight the results of our MHD model of flare-induced vertical kink oscillations excited by means of a lateral Gaussian-shaped pulse, modeling the effects of the kinetic energy release by the X 6.9-class solar flare.

Figure 3 shows the evolved perturbation in terms of plasma velocity, perturbed plasma density (defined as $\Delta \rho=[\rho(t)-$ $\rho(0)] / \rho(0)$, and the magnetic field in the $x z$ plane. We captured a snapshot at $t=14.7 \tau_{\mathrm{A}}$, at $y=0$, where $x$ is the injection direction of the velocity pulse. The form of the velocity pulse and the associated compression of the density are shown in Figs. 3a-b. The current density associated with the fast magnetosinic wave front produced by the pulse, and the corresponding changes of the magnetic field direction are evident in Figs. $3 \mathrm{c}-\mathrm{d}$, respectively. The response of the curtain produces velocity in the 


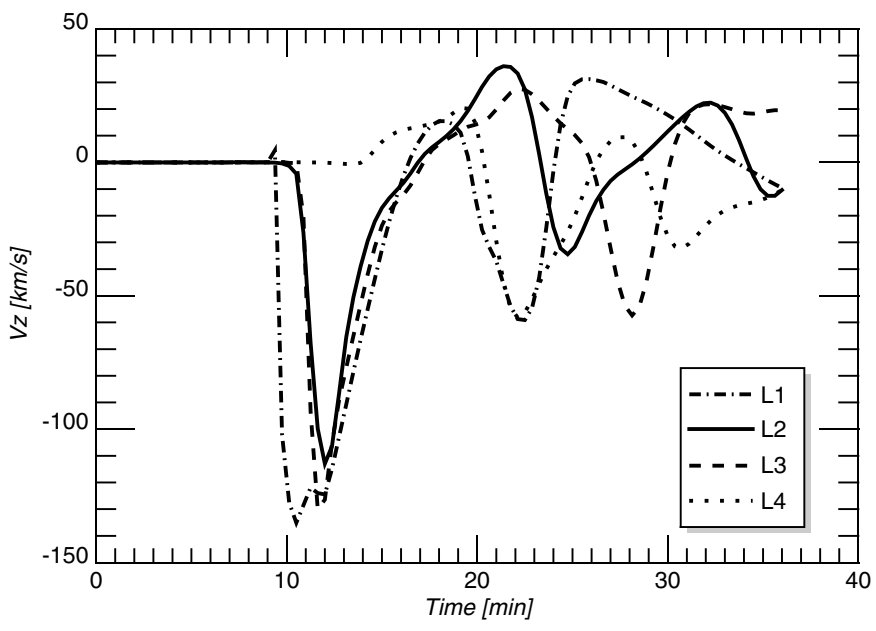

Fig. 4. Temporal evolution of the model velocity fluctuations in the $y z$ plane at different locations along the $x$ axis. L1: near flare blast deep layer oscillations $(-1.75,-3.35,1.15)$; L2: near apex deep layer oscillations $(0.0,1.7,1.3)$; L3: near apex surface oscillations $(0.0,1.7,1.85)$; L4: southward surface oscillations $(1.75,-0.9,1.65)$.

negative $x$ direction due to reflection and the restoring Lorentz force of the perturbed dipole field (see Fig. 3a) with a smaller magnitude than the pulse. The Gaussian pulse launched at the boundary causes rarefaction of the adjacent density region and propagation of a nonlinear (steepened) compressive fast magnetosonic wave which perturbs the magnetic structure of the curtain inducing oscillations nonlinearly. The propagation and the reflection of the wave is nonuniform because of the bipolar structure of the magnetic field and the corresponding structure of the fast magnetosonic speed (shown in Fig. 2b). As the fast magnetosonic wave leaves the computational domain, the oscillations of the curtain proceed to relax approaching a normal mode, and eventually damp as the transverse waves leak out of the structure, and the final density and magnetic field structure return close to the initial structure.

In Fig. 4, we show the temporal evolution of vertical fluctuations in the vertical plasma velocity component $V_{z}$ at locations L1-L4, which is very similar to what has been shown by observations (Fig. 1). We observe variations in the wave phase speed at different points, not necessarily aligned with the $y$ axis. The reason for this choice of "observing points" in the model comes from direct observations of the event by SG13, suggesting that the bundle of loops experiencing the vertical kink oscillations might be tilted to the bottom boundary surface and the oscillations are not necessarily coplanar.

The dash-dotted curve in Fig. 4 shows vertical transverse kink oscillations near the boundary plane where the flare produced pulse is modeled, i.e., nearest to the flaring site (L1) and taking place in the deeper layers of the plasma curtain $(z=1.3$, $\sim 20 \mathrm{Mm}$ above the photosphere). This oscillation has been interpreted by SG13 as the first overtone with a period of 8.9 min (cf. Fig. 1c for velocity time dependence). For oscillations in the northward side of the plasma curtain, our MHD model yields a fundamental period $P_{0}=2 \pi / \omega=9.2 \mathrm{~min}$ with high statistical confidence level, in surprisingly good agreement with SDO/AIA observations, given the simplicity of the present magnetic field model.

The solid curve in Fig. 4 shows the plasma vertical velocity versus time at locations L2 and the dashed curved shows L3: near the loop apex and midway from the flaring site $(y=0)$, respectively. In this region there is observational evidence for oscillations in both deep and surface layers of the coronal curtain ( $\sim 20 \mathrm{Mm}$ and $\sim 60 \mathrm{Mm}$ over the surface). According to SG13, the large-scale disturbance at depth could represent the fundamental kink mode (but, possibly dominated by the first harmonic, see below) with a period of $13.3 \mathrm{~min}$, while the nondecaying surface disturbance only perturbs the magnetic upper sheet of the curtain and is characterized by a period of $14.9 \mathrm{~min}$. Once again our MHD model of the event seems to reproduce the amplitude and period of the perturbations with a very good agreement: $A=34.8 \mathrm{~km} \mathrm{~s}^{-1}, P_{0}=13.5 \mathrm{~min}$ for the deep mode; $A=40.1 \mathrm{~km} \mathrm{~s}^{-1}, P_{0}=14.8 \mathrm{~min}$ for the surface mode, where the periods are obtained from the periodogram analysis (Scargle 1982), and the amplitude is obtained from sinusoidal least-squares fit with close values of the periods. The time profile of associated observed velocity perturbations can be seen in Fig. 1b.

Lastly, we display perturbations in the top layers of the coronal curtain, L4, shown with short dashes in Fig. 4, southward and opposite to the flaring site $(y=1.75)$. Vertical kink modes have been observed in this region as well, with a period of $12.7 \mathrm{~min}$ determined by SG13. Analysis of our modeled oscillations at this location yields $A=46.1 \mathrm{~km} \mathrm{~s}^{-1}$ and $P_{0}=11.7 \mathrm{~min}$, in good agreement with observational measurements (cf. Fig. 1b). The detection of the fundamental and second harmonic in the oscillations is important since their ratio in a coronal loop can be used for the determination of the gravitational scale height of the density (e.g., Andries et al. 2005; Ruderman et al. 2008; Verth et al. 2008). This effect is expected to be significant in long stratified coronal loop structures. However, in the present study we deal with oscillations of a bipolar structure in short magnetic field line loops (see Fig. 2), therefore, the magnitude of the second harmonic is found to be weak. Guided by SG13 observations, the high-contrast density structure of individual loops is not included in the model of the curtain.

In Fig. 5 we show the results of least-squares, exponentially damped, single-frequency sinusoidal fit to the modeled oscillation at L2. The fitted function is of the form

$v(t)=v_{0} \mathrm{e}^{-t / \lambda} \sin (\omega t+\phi)$,

where $\lambda$ is the damping time and $\omega=2 \pi / P$ is the oscillation frequency. We also show an undamped single-frequency sinusoidal fit. The damped sinusoidal fits produces a period of $12.8 \pm 0.8 \mathrm{~min}$ and the damping time in the range $6.2-11.7 \mathrm{~min}$. The fit produces an amplitude of $355 \mathrm{~km} \mathrm{~s}^{-1}$ and the phase $\phi=4.6 \mathrm{rad}$ at $t=0$. However, the values of the amplitude and the phase at $t=0$ are not directly relevant in this analysis, since the oscillations at L2 start at $t \sim 9 \mathrm{~min}$ because of the travel time of the pulse from the excitation boundary to L2. Because of the nonlinearity in the initial stages of the oscillations that produces large initial velocity fluctuation, the fitted exponential damping time is overestimated. However, the damping time is of the same order as the period of the oscillations. Since the large amplitude of the initial nonlinear pulse may affect the damped sinusoidal fit, we have also fitted undamped sinusoidal to the modeled velocity oscillations. At point L2 we get a period of $12.4 \pm 0.8 \mathrm{~min}$ for the undamped sinusoidal. The values of the periods obtained from the damped sinusoidal, undamped sinusoidal, and the periodogram analysis are close within the respective error bars.

The periodogram analysis (Scargle 1982) of the modeled oscillation time sequences for points L2 and L3 are shown in Fig. 6. It is evident that the oscillations are dominated by a single frequency with similar results at points L1 and L4 (not shown). While there is some evidence of second and higher harmonics in the analysis, the statistical confidence level of these harmonics is 
Table 1. Comparison between observed and model oscillation periods for the four detected locations in SG13.

\begin{tabular}{lcccc}
\hline \hline Location of observation & Observed periods [min] & MHD period [min] & $B_{\mathrm{MHD}}(\mathrm{G})$ & $B_{\mathrm{CS}}(\mathrm{G})$ \\
\hline Flare Blast (L1) & 8.9 & 9.2 & 2.2 & 8.4 \\
Apex Inside (L2) & 13.3 & $13.5^{*}$ & 3.1 & 4.3 \\
Apex Surface (L3) & 14.9 & 14.8 & 2.9 & 4.3 \\
Southward Surface (L4) & 12.7 & 11.7 & 3.4 & 4.9 \\
\hline
\end{tabular}

Notes. The values of the MHD periods were obtained using periodogram analysis (Scargle 1982) and the period with the highest intensity was chosen as the proxy for the normal mode. The penultimate column shows the magnetic field strength obtained from the 3D MHD model, and the last column is from simplified coronal seismology (CS). ${ }^{(*)}$ At point L2 the average of two periods near the peak are given since they have close high confidence level (see Fig. 6).

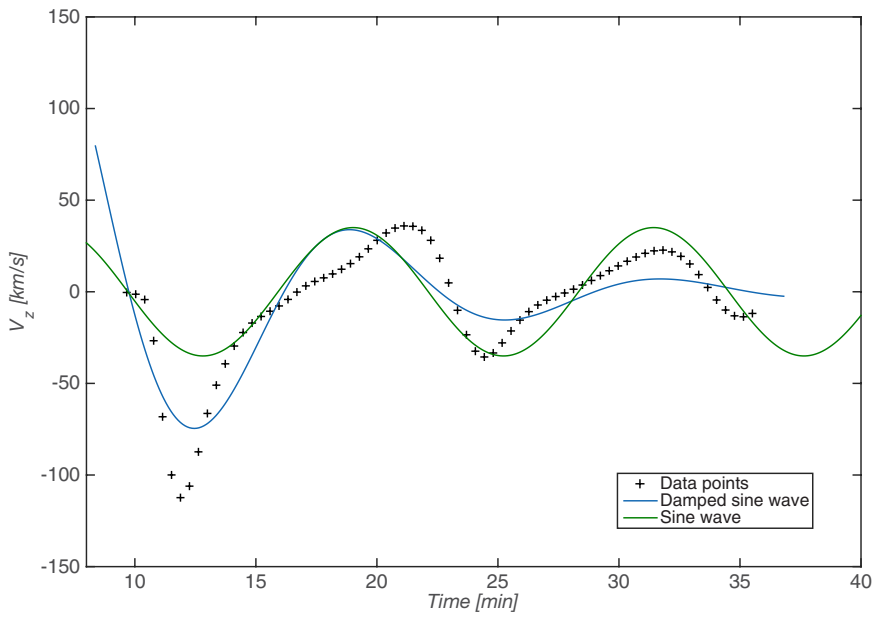

Fig. 5. Temporal evolution of the model velocity oscillation in the $y z$ plane at L2 ("+" symbols) fitted with a single frequency sinusoidal function (green) and the damped sinusoidal function (blue). The resulting period for the damped sinusoidal is $12.8 \pm 0.8 \mathrm{~min}$ and the best-fit damping time is in the range $6.2-11.7 \mathrm{~min}$. The best-fit period is $12.4 \pm 0.8 \mathrm{~min}$ for the sinusoidal fit.

low $<1 \sigma$. The low statistical significance of the second harmonic may explain why the second harmonic is rarely detected in observations of loop oscillations. We also performed curve fitting of single-period sinusoidal and exponentially damped sinusoidal function to the oscillations and found that the values of the fitted periods are in excellent agreement with the periodogram values. We find that the damping time of the oscillations is on the order of the oscillation period.

Table 1 summarizes our results from the model velocity oscillation, and compares them to SG13 results obtained using SDO/AIA data. The modeled error bar of the periods obtained from least-square fitting is below $3 \%$. However, since there is no statistical variability in a computational model, the meaning of the error bars is ill-defined in this context. Therefore, the computational values are shown without error bars. The last two columns of Table 1 show the comparison between the mean magnetic field along the magnetic field lines (loops) that pass through the measurement points in the 3D MHD model ( $\left.B_{\mathrm{MHD}}\right)$, and the magnetic field inferred from application of coronal seismology $(\mathrm{CS}), B_{\mathrm{CS}}$. Since the gravitationally stratified density and the magnetic field vary significantly along the loops (e.g., Ofman et al. 2012a), we use the average values along the magnetic loops. We are comparing the values of the average magnetic field along the magnetic loops that pass through the points L1-L4 in our MHD model, with the average value of the magnetic field determined from coronal seismology that uses a slab model for these loops (Eq. (4)). Since the density along a loop is close to

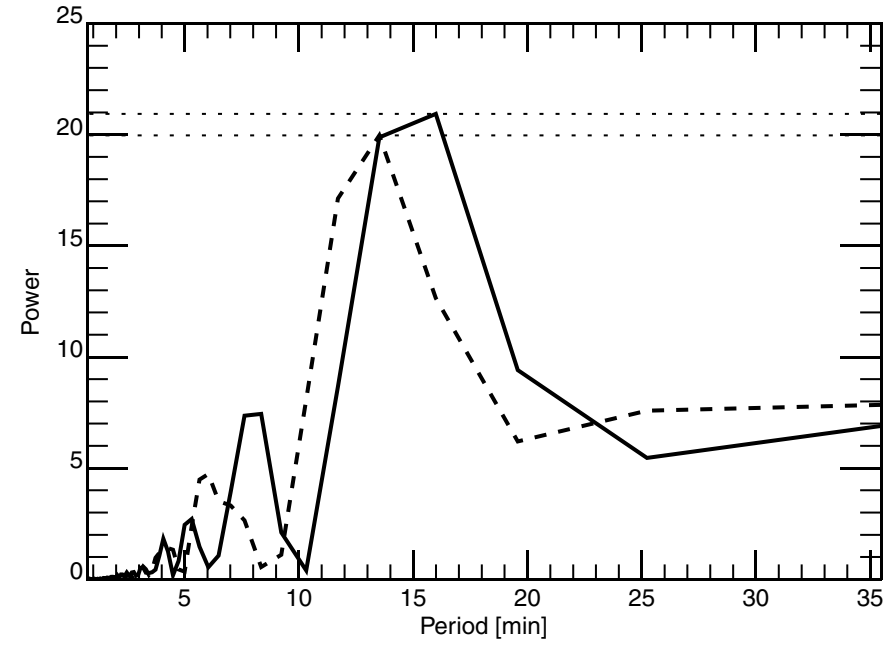

Fig. 6. Results of the periodogram analysis (Scargle 1982) of the oscillations at L2 (solid) and L3 (dashes). The confidence level of the dominant period is shown with the dashed line for each periodogram.

the surrounding density, we use $C_{k} \approx V_{\mathrm{A}}$ in the low- $\beta$ plasma, and we calculate the loop-averaged density to find $B_{\mathrm{CS}}$. Since the wavelength of the fundamental mode oscillations is twice the loop length, the waves cannot be used to determine magnetic field value at a particular point of the variable magnetic field in the loop (see Fig. 3 in Ofman et al. 2012a, for an example of magnetic field, density, and fast magnetosonic speed variability along a loop in the bipolar field model). The long wavelength oscillations only provide information on the average magnetic field in thes loops, and these values are given in Table 1.

The event animation from SDO/AIA shows how the plasma curtain oscillations are combinations of the fundamental and second normal modes. However, the latter contribution seems to be predominant at deep and surface levels. Therefore, CS predictions are computed using the expression for second harmonic oscillations of the ideal vertical kink mode without density contrast across the loop (consistent with observations that show little density contrast), and average values along the loops

$B_{\mathrm{CS}}=\langle\rho\rangle^{1 / 2} \frac{L}{P_{0}}$,

where $\langle\rho\rangle$ is the average density of the loop, $L$ is the loop length, $P_{0}$ is the oscillation period in the MHD model, and $B_{\mathrm{CS}}$ is the average magnetic field magnitude along the loop.

The periods of the observed and modeled oscillations show good agreement. Comparing the MHD and coronal seismology predictions of the mean magnetic field, we conclude that the differences are within $39-47 \%$ at three out of four observation locations (Figs. $4 b-d$ and row $2-4$ in Table 1). This level of 
agreement was also found by Aschwanden \& Schrijver (2011) when comparing $B_{\mathrm{CS}}$ values to extrapolated magnetogram field values and by Pascoe \& De Moortel (2014) in 3D MHD arcade model test of CS. The poorer agreement between the values near the flare blast location (Fig. 4a and row 1 in Table 1, $B_{\mathrm{CS}} / B_{\mathrm{MHD}} \approx 4$ ) is likely due to two factors: the large tilt of the magnetic loop that passes through the observation point to the $y z$ plane, causing the loop to interact with the lower boundary surface; and the possibility that the observed oscillation is not a normal harmonic, but a driven propagating mode due to the close proximity of the boundary that launches the Gaussian velocity pulse.

\section{Discussion and conclusions}

The article demonstrates that an ideal dipole approximation of the magnetic field geometry is a good approximation of the magnetic field structure in an AR plasma curtain on a large spatial scale over the western equatorial corona observed on 2011 August 9 by SDO/AIA. This large-scale magnetoplasma system, when encountered with the flare generated propagating disturbances, produces multiple harmonics of vertical kink oscillations in different parts of the curtain as well as surface oscillations. The 3D MHD numerical simulation domain includes a magnetoplasma system with gravitationally stratified density, and its interaction with the velocity pulse introduced at the boundary mimicking the flare disturbance, which propagates into the modeled AR curtain and produces magnetic field displacement and velocity oscillations of vertical kink waves. The results of the numerical simulation match well with the observations of SG13, while it is initialized with parameters based on the observed conditions, and thus improve the understanding of the wave activity.

While the present 3D MHD model produces the overall evolution of the vertical transverse waves in good agreement with observations, the scope of the model is limited, and is not intended to study flares and the detailed heating and cooling dynamics of active regions. The main limitation of the model is the use of dipole field that is only approximately applicable to this observation, and this could be improved using extrapolated magnetogram field (e.g., Ofman 2007) in future studies. The detailed effects of coronal heating, thermal conduction, and radiative cooling are not modeled and only approximately accounted for. The use of a polytropic energy equation with $\gamma=1$ is only applicable to approximately isothermal conditions where the above effects nearly balance and the heat conduction is high along the field. The initially hydrostatic density structure, while providing overall reasonable approximation, does not capture the full complexity of the active region and individual loop density structure and neglects the potentially important role of upflows and downflows that can affect the wave dynamics.

The damping of the oscillations in the model is due to refraction and leakage of the fast mode wave flux, as demonstrated in previous work (Ofman \& Thompson 2002; McLaughlin \& Ofman 2008; Selwa \& Ofman 2009; Selwa et al. 2011a), since nonideal damping processes, such as resistive or numerical dissipation, are small in the present model. It is evident that the observed oscillations appear to damp on a timescale similar to the modeled evolution. The effects of mode conversion (i.e., resonant absorption), as discussed by SG13, are possible but not strongly supported since the observed AR curtain shows little evidence of the individual loop structures (with high density contrast) necessary for this damping mechanism. The modeled low- $\beta$ plasma curtain contains a strong magnetic field and the density is gravitationally stratified to mimic the observations and to best fit with the realistic solar atmosphere. Therefore, a significant fast-mode speed gradient is set across the plasma curtain from its deeper layers toward the apex. This may produce internal refraction of the waves and transfer of the fast mode (kink) wave energy toward the surface and outside the curtain (as demonstrated numerically by, e.g., Ofman \& Thompson 2002). An alternative interpretation of the refraction process can be found in Goossens et al. (2002, 2011, 2012), adapted by SG13 initial analysis.

In this study, we demonstrate the significant advancement of coronal seismology by applying more realistic magnetic field topology and density model in 3D MHD calculation improving on application of slab or cylindrical linear modes, while not necessarily reproducing the details of actually observed coronal active region. The 3D MHD model of the waves in the coronal curtain allows us to test the application of simplified coronal seismology, as it is widely used (see the reviews, e.g., Nakariakov \& Verwichte 2005; Liu \& Ofman 2014) to the present model and compare the results of CS with the actual values of the magnetic field used in the 3D MHD model. We find that simplified coronal seismology provides magnetic field values within $40 \%-50 \%$ of the actual values. Noting that the magnetic energy of AR important for space weather impact analysis scales as $B^{2}$, this discrepancy amounts to a factor of $\sim 2$ of the magnetic energy content. This result is consistent with previous tests of CS in more simplified setup (e.g., Pascoe \& De Moortel 2014, in magnetic arcade without gravity) and with comparisons to extrapolated magnetogram field (Aschwanden \& Schrijver 2011). Thus, to improve CS and provide more accurate values of the magnetic field in ARs it is necessary to consider the properties of waves in complex, curved, and more realistic magnetic structures with realistic global density structure as demonstrated in the present study.

Acknowledgements. We are grateful to SDO/AIA team for providing the data used in this study. L.O. was supported by NASA grants NNX11AO68G and NNX12AB34G. A.K.S. acknowledge the patient encouragements of Shobhna. We thank T.J. Wang for useful discussions.

\section{References}

Al-Ghafri, K. S., Ruderman, M. S., Williamson, A., \& Erdélyi, R. 2014, ApJ, 786,36

Andries, J., Goossens, M., Hollweg, J. V., Arregui, I., \& Van Doorsselaere, T. 2005, A\&A, 430, 1109

Andries, J., van Doorsselaere, T., Roberts, B., et al. 2009, Space Sci. Rev., 149, 3

Aschwanden, M. J. 2005, Physics of the Solar Corona, An Introduction with Problems and Solutions, 2nd edn. (Springer-Praxis)

Aschwanden, M. J., \& Schrijver, C. J. 2011, ApJ, 736, 102

Aschwanden, M. J., Fletcher, L., Schrijver, C. J., \& Alexander, D. 1999, ApJ, 520,880

De Moortel, I., \& Nakariakov, V. M. 2012, Royal Soc. London Phil. Trans. Ser. A, 370, 3193

Erdélyi, R., Al-Ghafri, K. S., \& Morton, R. J. 2011, Sol. Phys., 272, 73

Goossens, M., Andries, J., \& Aschwanden, M. J. 2002, A\&A, 394, L39

Goossens, M., Erdélyi, R., \& Ruderman, M. S. 2011, Space Sci. Rev., 158, 289

Goossens, M., Andries, J., Soler, R., et al. 2012, ApJ, 753, 111

Gruszecki, M., Murawski, K., Selwa, M., \& Ofman, L. 2006, A\&A, 460, 887

Kim, S., Nakariakov, V. M., \& Cho, K.-S. 2014, ApJ, 797, L22

Lemen, J. R., Title, A. M., Akin, D. J., et al. 2012, Sol. Phys., 275, 17

Liu, W., \& Ofman, L. 2014, Sol. Phys., 289, 3233

McIntosh, S. W., de Pontieu, B., Carlsson, M., et al. 2011, Nature, 475, 477

McLaughlin, J. A., \& Ofman, L. 2008, ApJ, 682, 1338

Miyagoshi, T., Yokoyama, T., \& Shimojo, M. 2004, PASJ, 56, 207

Morton, R. J., \& Erdélyi, R. 2009, ApJ, 707, 750

Nakariakov, V. M., \& Verwichte, E. 2005, Liv. Rev. Sol. Phys., 2, 3

Nakariakov, V. M., Ofman, L., DeLuca, E., Roberts, B., \& Davila, J. M. 1999, Science, 285,862 
L. Ofman et al.: 3D MHD modeling of vertical kink oscillations in a plasma curtain

Nisticò, G., Nakariakov, V. M., \& Verwichte, E. 2013, A\&A, 552, A57

Nisticò, G., Anfinogentov, S., \& Nakariakov, V. M. 2014, A\&A, 570, A84

Ofman, L. 2007, ApJ, 655, 1134

Ofman, L. 2009, ApJ, 694, 502

Ofman, L., \& Thompson, B. J. 2002, ApJ, 574, 440

Ofman, L., Wang, T. J., \& Davila, J. M. 2012, ApJ, 754, 111

Pascoe, D. J., \& De Moortel, I. 2014, ApJ, 784, 101

Powell, K. G. 1994, Report NM-R9407

Ruderman, M. S. 2011, A\&A, 534, A78

Ruderman, M. S., Verth, G., \& Erdélyi, R. 2008, ApJ, 686, 694

Scargle, J. D. 1982, ApJ, 263, 835

Schmidt, J. M., \& Ofman, L. 2010, ApJ, 713, 1008

Selwa, M., \& Ofman, L. 2009, Annales Geophysicae, 27, 3899

Selwa, M., Murawski, K., Solanki, S. K., Wang, T. J., \& Tóth, G. 2005, A\&A, 440,385

Selwa, M., Solanki, S. K., Murawski, K., Wang, T. J., \& Shumlak, U. 2006, A\&A, 454, 653

Selwa, M., Murawski, K., Solanki, S. K., \& Wang, T. J. 2007, A\&A, 462, 1127
Selwa, M., Ofman, L., \& Solanki, S. K. 2011a, ApJ, 726, 42 Selwa, M., Solanki, S. K., \& Ofman, L. 2011b, ApJ, 728, 87

Selwa, M., Poedts, S., \& DeVore, C. R. 2013, Sol. Phys., 284, 515

Srivastava, A. K., \& Goossens, M. 2013, ApJ, 777, 17

Van Doorsselaere, T., Wardle, N., Del Zanna, G., et al. 2011, ApJ, 727, L32

Verth, G., Erdélyi, R., \& Jess, D. B. 2008, ApJ, 687, L45

Verwichte, E., Foullon, C., \& Nakariakov, V. M. 2006, A\&A, 452, 615

Verwichte, E., Van Doorsselaere, T., Foullon, C., \& White, R. S. 2013a, ApJ, 767,16

Verwichte, E., Van Doorsselaere, T., White, R. S., \& Antolin, P. 2013b, A\&A, 552, A 138

Wang, T. J., \& Solanki, S. K. 2004, A\&A, 421, L33

Wang, T. J., Solanki, S. K., \& Selwa, M. 2008, A\&A, 489, 1307

Wang, T., Ofman, L., Davila, J. M., \& Su, Y. 2012, ApJ, 751, L27

Wang, T., Ofman, L., \& Davila, J. M. 2013, ApJ, 775, L23

White, R. S., \& Verwichte, E. 2012, A\&A, 537, A49

White, R. S., Verwichte, E., \& Foullon, C. 2012, A\&A, 545, A129

Yuan, D., \& Nakariakov, V. M. 2012, A\&A, 543, A9 